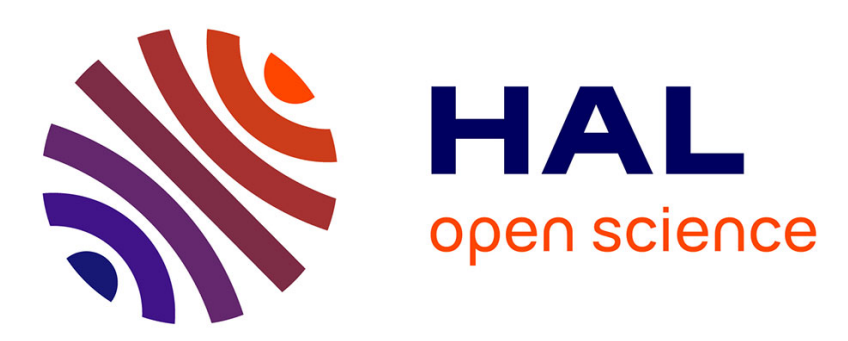

\title{
Atelier d'écriture et troubles du comportement à l'adolescence: fonction contenante des cadres en thérapie à médiation avec un adolescent placé
} Corentin Boulay, Nadine Demogeot, Joëlle Lighezzolo-Alnot

\section{- To cite this version:}

Corentin Boulay, Nadine Demogeot, Joëlle Lighezzolo-Alnot. Atelier d'écriture et troubles du comportement à l'adolescence: fonction contenante des cadres en thérapie à médiation avec un adolescent placé. La psychiatrie de l'enfant, 2020, 63, pp.197-217. 10.3917/psye.631.0197 . hal-02357264

\author{
HAL Id: hal-02357264 \\ https://hal.science/hal-02357264
}

Submitted on 31 May 2021

HAL is a multi-disciplinary open access archive for the deposit and dissemination of scientific research documents, whether they are published or not. The documents may come from teaching and research institutions in France or abroad, or from public or private research centers.
L'archive ouverte pluridisciplinaire $\mathbf{H A L}$, est destinée au dépôt et à la diffusion de documents scientifiques de niveau recherche, publiés ou non, émanant des établissements d'enseignement et de recherche français ou étrangers, des laboratoires publics ou privés. 


\title{
ATELIER D’ÉCRITURE ET TROUBLES DU COMPORTEMENT
}

À L'ADOLESCENCE : FONCTION CONTENANTE DES CADRES

\author{
EN THÉRAPIE À MÉDIATION AVEC UN ADOLESCENT PLACÉ
}

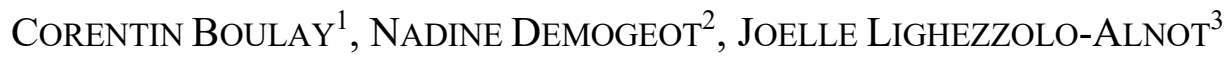

\begin{abstract}
ATELIER D'ÉCRITURE ET TROUBLES DU COMPORTEMENT À L'ADOLESCENCE : FONCTION CONTENANTE DES CADRES EN THÉRAPIE À MÉDIATION AVEC UN ADOLESCENT PLACÉ
\end{abstract}

La littérature d'obédience psychanalytique nous indique que les troubles du comportement à l'adolescence sont souvent liés à un défaut de la fonction contenante du psychisme. À cet égard, la proposition thérapeutique doit pouvoir agir en contrepoint de ces organisations du psychisme pour favoriser la reprise des processus de contenance. L'objectif de cet article vise à étudier le fonctionnement d'une médiation thérapeutique par l'écriture auprès d'un adolescent en situation de placement. Á l'appui de données cliniques recueillies dans le cadre d'une psychothérapie d'adolescent, nous avons procédé à une analyse des processus de symbolisation mobilisés dans le cadre d'un atelier à médiation par l'écriture. Les résultats nous indiquent que la médiation favorise l'émergence de la fonction contenante à l'appui de la multiplicité des cadres qui prennent place dans la médiation écriture, et qu'à partir de cette fonction peut s'étayer l'amorce d'un travail de représentation. Les médiations par l'écriture semblent ainsi adaptées au travail thérapeutique avec des adolescents placés, et il serait pertinent de développer des recherches centrées sur l'évaluation de leurs effets.

Mots-clés : Médiations thérapeutiques, écriture, adolescence.

Pour citer cet article : Boulay, C., Demogeot, N. Lighezzolo-Alnot, J. (2020). Atelier d'écriture et troubles du comportement à l'adolescence : fonction contenante des cadres en thérapie à médiation avec un adolescent placé. La psychiatrie de l'enfant, 63 (1), 151-195. DOI : $10.3917 /$ psye. 631.0197

\section{INTRODUCTION}

Adolescence, placement et troubles du comportement

Il est important que les adolescents accompagnés et placés par l'Aide Sociale à l'Enfance (ASE) fassent l'objet d'une attention particulière, étant donné qu'ils constituent une population à haut risque de développer des troubles psychiatriques. Pathologies de l'agir, violence et conduites à risque, les symptômes externalisés sont extrêmement répandus chez cette population, qui représente la majorité des demandes de consultation en psychiatrie pour des troubles des conduites ou comportements oppositionnels (Chatagner et al., 2015). Ces adolescents sont

\footnotetext{
${ }^{1}$ Doctorant en psychologie, Université de Lorraine, laboratoire Interpsy, EA 4432.

${ }^{2}$ Maître de conférences en psychologie clinique, Université de Lorraine, laboratoire Interpsy, EA 4432. ${ }^{3}$

Professeure de psychologie clinique, Université de Lorraine, laboratoire Interpsy, EA 4432.
} 
également plus concernés par les morbidités psychiatriques que leurs homologues placés en familles d'accueil ou bien vivant au domicile familial (Vitte et al., 2017). Kayser et al. (2011) ont proposé que ces phénomènes psychopathologiques puissent être liés à l'exposition durable de ces jeunes à un certain nombre de facteurs de risques : maltraitances, conflits familiaux, négligences, précarité sociale, auxquels s'ajoutent des problématiques de ruptures successives et répétées avec la famille ou les lieux de placement (O’Neil et al., 2012).

$\mathrm{Au}$ regard de ces facteurs de risques, la souffrance des adolescents placés apparaît s'originer dans des parcours chaotiques, des environnements familiaux fragilisant et fragilisés, caractérisés par la défectuosité des liens précoces (Chatagner et al., 2015). De ce point de vue, les problématiques de placement et leur cortège de troubles externalisés traduisent également des difficultés liées à la construction des liens d'attachement : les sujets en situation de placement développent plus souvent des configurations d'attachements insécurisées qui impactent leur développement affectif et cognitif (Lemoust de Lafosse et Blanc, 2016). Ces relations précoces dysfonctionnelles et leurs conséquences au niveau des processus d'attachement mettent en évidence les limites des premiers mouvements de subjectivation, construits autour de la fonction contenante portée par l'environnement.

\section{Fonction contenante et représentation}

Cette fonction psychique joue un rôle primordial dans la construction de la subjectivité, car elle est le socle sur lequel va pouvoir s'installer la capacité du sujet à se protéger contre les menaces qui émanent de l'extérieur. Cette fonction de pare-excitation psychique permet la construction de la capacité à représenter, donc à mettre en forme les perceptions afin de les rendre compréhensibles par le psychisme. Le travail de subjectivation et de construction du monde interne, l'apparition de la vie psychique, passent par l'établissement premier de la fonction contenante.

Les premiers caregivers ont pour fonction, notamment par le biais des activités de holding et d'accordages, par leur capacité de rêverie, de proposer un espace de contenance au sein duquel sera rendue possible l'existence des contenus psychiques et les processus de transformation des éléments a-signifiants de l'environnement en éléments de sens intégrables par le sujet (Bion, 1962).

La subjectivité se forme alors autour d'un premier processus d'intériorisation de la fonction contenante de l'environnement, qui donne forme et structure encadrante au moi (Haag, 2005). Le psychisme acquiert la capacité à mettre en représentation son expérience subjective, à construire et mettre en forme des contenus de pensée signifiants et représentants intériorisés de la perception (Chouvier, 2003 ; Haag, 2005). Ce mouvement d'intériorisation de la contenance construit le psychisme, qui devient à la fois structure contenante du moi et représentation de contenance. L'intériorisation de la fonction contenante fournie par l'environnement rend possible le double mouvement de construction psychique entre acquisition de la structure (contenant) et de la représentation (contenu) (Golse, 2007). La toile de fond du psychisme sur laquelle vont prendre forme les représentations s'étaye sur un premier travail de représentation lié à la figure de l'absence : la symbolisation initiale de l'absence de représentation favorise l'intégration de la représentation de l'absence, qui soustend la constitution du fond psychique (Rabeyron, 2016).

Ces processus primaires à l'origine de la pensée sont les prérequis à deux formes de capacités psychiques : 1/ la capacité d'être seul (Green, 1993 ; Winnicott, 1958) qui marque la possibilité d'existence psychique hors de la dépendance à la présence effective de l'autre, donc l'intériorisation de la représentation de l'absence qui favorise l'arrivée de la représentation de 
l'objet absent ; 2/ la fonction réflexive qui vise à permettre l'autoinformation de la psyché sur son fonctionnement. Lorsque le sujet se trouve dépourvu de cette fonction contenante principale, le psychisme qui ne s'est pas suffisamment construit autour de la capacité à représenter se voit contraint de trouver des solutions « bio-logiques » (Roussillon, 1999a) face aux situations d'adversité, c'est à dire à des solutions ancrées dans le corps et la perception, faute de capacité suffisante à représenter et donc à mobiliser l'espace psycho-logique, celui du signifiant et du symptôme. C'est alors qu'apparaissent les processus primaires traumatiques, caractérisés par des sentiments indicibles témoins de l'effacement du sujet (Aulagnier, 1975), et dont les troubles du comportement peuvent être le signe chez les adolescents placés.

\section{Adolescence et maltraitances infantiles}

Ces sujets peuvent donner à voir les angoisses primitives, originaires, liées à leurs expériences traumatiques (Derivois, 2008) qui rendent compte de l'échec de la mise en représentation de leur histoire. Les troubles de l'adolescent en situation de placement sont ainsi fortement liés à des expériences traumatiques antérieures (Drieu et al., 2012), à partir desquels la fonction contenante a pu être mise en défaut. La construction subjective de ces adolescents s'organise autour des limites d'un environnement précoce insuffisamment disponible, qui contrarie l'intériorisation de sa fonction contenante (Green, 1993) par le psychisme.

L'absence d'étayage de l'environnement, insuffisamment disponible ou au courant des besoins du sujet en développement, a pour conséquence de laisser l'enfant dans un état traumatique favorisant le clivage et le blocage des processus d'intégration des phénomènes perceptifs. En ce sens, ces « terreurs sans noms » (Bion, 1983) véhiculent une charge d'angoisse inépuisable et sans recours face à une perception qui ne peut s'inscrire comme une représentation. Nombreux sont les adolescents placés qui ont rencontré des maltraitances à l'origine des expériences agonistiques (Winnicott, 1974). Ces expériences peuvent avoir pour conséquences de couper le sujet de sa subjectivité par un processus de clivage protégeant l'intégrité du moi en rejetant hors de la conscience la perception traumatique. Le clivage et le retrait de la subjectivité constituent alors un terreau privilégié à l'apparition ultérieure de phénomènes de compulsions de répétition dans les situations de rappel de l'épisode traumatique initial. Lorsque la perception est réactualisée chez un sujet qui ne dispose pas des capacités représentatives pour mettre en sens cet événement, il se retrouve contraint à déployer un ensemble de stratégies visant à tenter de trouver des solutions d'intégration (compulsion de répétition), qui dans le même temps témoignent de l'échec de solutions relatives à l'intégration psychique de l'événement traumatique. Ces solutions sont alors orientées vers des comportements de retrait de la subjectivité, visant à éloigner la perception de la conscience, ou bien du côté de l'agir violent qui consiste en la projection sur la réalité externe des contenus en attente de symbolisation (Houssier, 2008).

\section{Le pubertaire : organisation et réactualisation de la pulsion}

Le pubertaire est un moment d'intégration des nouveaux conflits psychiques, en lien avec la maturation corporelle et les possibilités de satisfaction pulsionnelle qu'elle permet. La puberté et le développement des caractères sexuels, qui font sortir l'adolescent de la période de latence, rendent réalisables les motions pulsionnelles contre lesquelles le psychisme avait dressé les barrières surmoïques du refoulement. L'enjeu de l'adolescence concerne alors la découverte de nouveaux modes de symbolisation d'une pulsion qui devient réalisable (Roussillon, 1999b). En ce sens, ce qui change à l'adolescence concerne tant la nature de la pulsion et de sa satisfaction, que la nature de la fonction symbolisante du psychisme. Dans le même temps, c'est une 
succession de deuils que doit réaliser l'adolescent : celui de la position infantile, des liens œdipiens entretenus avec la famille, engageant également à un travail de symbolisation nécessaire à la poursuite de son développement. Enfin, l'enjeu de l'adolescence est aussi un enjeu de subjectivation et d'individuation (Birraux, 2013), qui en passe par l'épreuve des séparations (Chabert, 2013). L'adolescence est donc un temps de construction de l'identité et de symbolisation qui se fonde sur une succession de re-mobilisations et de transformations psychiques et corporelles, dont la résolution doit mener à l'autonomie psychique.

L'environnement joue encore une fois un rôle primordial dans la construction de l'adolescent (Braconnier, 2009). Il doit à nouveau exercer sa fonction contenante pour favoriser l'intégration des pulsions du pubertaire. L'entourage agit alors comme un «moi-auxiliaire » (Corcos, 2007) qui soutient le processus d'intégration de l'adolescent. En ce sens, sa stabilité sera une condition à l'intégration psychique des conflits de l'adolescence, car il agit à nouveau comme dans les premiers instants de l'enfance, à savoir comme un médium malléable qui va favoriser l'appropriation subjective du vécu de l'individu en proie aux pulsions de l'adolescence.

L'adolescence est également un espace de réactualisation des conflits infantiles irrésolus au cours du développement psycho-affectif, qui se déroule sur la scène familiale, re-mobilisant les fixations pulsionnelles parentales. À cet égard, nous pouvons interroger le statut de ces adolescents placés en institution, qui ont été très tôt confrontés aux expériences traumatiques et à l'absence de contenance environnementale, et qui par la suite se retrouvent face à des ruptures qui impactent la stabilité des environnements ultérieurs (placements, dé-placements, replacements...). Les « trous » dans la contenance familiale seraient alors des facteurs permettant d'appréhender l'émergence des troubles de l'adolescent (Hanifi et al., 2019). La complexité des situations familiales peut ainsi tendre à fragiliser le travail de construction de la subjectivité et compliquer le déroulement du processus adolescent.

L'immaturité, la faiblesse du moi et le manque de soutien environnemental peuvent faciliter l'émergence de conduites problématiques, externalisées, particulièrement autour de l'agir violent. Le recours à l'acte violent est souvent théorisé comme relevant de trois grands processus : il peut renvoyer à une problématique de décharge pulsionnelle ; à une tentative de défense contre ce qui menace l'identité ; il peut enfin être envisagé comme une tentative de liaison entre des éprouvés et des représentations déliées psychiquement (Chagnon et Houssier, 2012).

Dans chacun de ces cas, l'agir violent revêt en conséquence une fonction psychique spécifique. En ce sens, la violence à l'adolescence semble s'organiser comme une tentative d'intégration, une compulsion de répétition, des expériences non symbolisées. La violence et le passage à l'acte à l'adolescence peuvent alors désigner un phénomène de projection topique à l'extérieur du sujet, d'un conflit interne insoluble (Hanifi et al., 2019) et en attente de signification portée par l'environnement (Roussillon, 1999a). Le recours aux comportements violents peut ainsi s'expliquer du fait d'une immaturité psychique, en lien avec les limites dans la fonction contenante, réactualisée et perturbée par l'arrivée du traumatisme pubertaire (Corcos, 2007), et par le manque d'auxiliaire médiatisant les capacités de symbolisation du sujet.

\section{Le clinicien comme surface de projection : transfert et contre-transfert}

Le manque de structure contenante à l'adolescence et la problématique de répétition qu'elle implique favorisent la rupture de l'équilibre dans les investissements narcissiques et objectaux. Cette problématique débouche ainsi sur les phénomènes de projection, au cours desquels l'autre n'est pas encore investi sur un versant génitalisé, mais comme un moyen de transposition des conflits internes dans le monde perceptivo-moteur (Jeammet, 2002). C'est de cette manière que 
les comportements d'opposition peuvent prendre racine dans un environnement spécifique, comme un écho externalisé des conflits qui se déroulent au niveau psychique. C'est alors une répétition en acte qui prend place dans la dynamique transférentielle, au sein de laquelle l'objet se verra dépositaire des affects intolérables pour l'adolescent et à qui la tâche incombe de les rendre intégrables, comme un nouveau processus d'appropriation du monde de l'adolescent médiatisé par la capacité de rêverie de l'objet. La souffrance des adolescents, sous un versant externalisé, est donc également caractérisée par la difficulté qu'ils font éprouver aux autres (Botbol et al., 2010). En effet, la clinique avec des adolescents qui présentent des troubles du comportement place le clinicien dans une position particulière qui bouscule les codes des cadres psychothérapeutiques classiques, qu'il devient nécessaire de revisiter (Jeammet, 2019). La dynamique transféro-contre-transférentielle porte donc les marques des traumatismes précoces liés au sujet et demande au clinicien une capacité à se placer en objet (Corcos, 2007), en médium malléable (Milner, 1955), qui pourra tolérer et survivre à la tempête de la répétition pour en permettre la symbolisation (Chagnon et Houssier, 2012). Pour Jeammet (2001), les troubles du comportement demandent l'exercice d'un cadre contenant qui rend possible l'apaisement des conflits, la construction puis le maintien des liens. C'est ainsi au travers de la relation thérapeutique et des dispositions psychiques du thérapeute que le travail de médiation entre l'adolescent et ses éprouvés pourra s'engager.

Dans cette perspective, diverses questions se posent concernant la prise en charge des adolescents qui mettent en déroute les dispositifs cliniques du fait de manifestations comportementales par trop intenses : comment favoriser l'existence d'un cadre contenant lorsque le cadre interne et les cadres environnementaux se dérobent de manière incessante ? Comment permettre aux cliniciens d'élaborer des dispositifs qui tiennent compte des spécificités de ces adolescents et qui rendent possible l'exercice de la survivance de l'objet ? Et enfin, quels sont les éléments qui, dans les dispositifs cliniques, participent de la restauration du cadre interne?

\section{MÉTHODE}

\section{Médiations thérapeutiques par l'écriture}

$\mathrm{Au}$ regard de cet état des lieux, qui nous fournit une piste de compréhension de la souffrance des adolescents placés, nous identifions d'une part que l'axe central de la problématique de cette population se situe au niveau de l'instauration de la fonction contenante du psychisme, et d'autre part que les « ingrédients » essentiels à la restauration de ces fonctions, en contexte psychothérapeutique, reposent sur la survivance de l'objet-clinicien et sur la malléabilité du cadre thérapeutique. Par ailleurs, la question du pubertaire ne peut être mise de côté, malgré les fixations infantiles certaines qui empêchent la construction psychique de ces adolescents. La proposition thérapeutique doit alors porter à la fois la marque des expériences primitives non symbolisées et celle de la construction identitaire et de l'élaboration de la pulsion sexuelle propre à la conflictualité de l'adolescence.

Pour explorer ces questions, nous avons envisagé de proposer un atelier à médiation par l'écriture à l'attention d'adolescents confiés à l'Aide Sociale à l'Enfance et placés dans un foyer d'accueil. En effet, le champ thérapeutique des médiations et l'exploration des ateliers d'écriture proposent des outils favorables à la problématique soulevée par les adolescents confiés. Ces dispositifs cliniques, d'orientation psychanalytique, reposent sur l'articulation des processus d'associativité et de transfert, appliqués au processus de manipulation d'un matériau (médium malléable), support de projection de la représentation de soi, de l'autre, de la relation. La manipulation du matériau, ses caractéristiques et l'interaction avec le clinicien rendent 
possible la mise en forme des expériences psychiques non symbolisées (Brun, 2014). La médiation a alors pour fonction de mobiliser le corps et avec lui les processus primaires de la symbolisation. En ce sens, la médiation se veut appropriée au travail de reprise des expériences archaïques non symbolisées en reposant sur l'associativité sensori-motrice, au sein de laquelle la matière malléable et le processus créateur représentent la matière et les processus psychiques.

Les travaux de Brun (2014) nous éclairent sur les processus qui opèrent au sein d'une thérapie à médiation. Un premier mouvement de projection topique (Brun et al., 2013) sur le médium permet la réalisation d'un transfert de la problématique psychique de l'individu sur la matière sensible et au sein du cadre thérapeutique lui-même. Cette configuration transférentielle comporte alors les formes primaires de symbolisation non intégrables, les mouvements de destructivité, les atteintes à l'objet, qui disent en miroir l'expérience subjective du sujet. À partir de cette expérience transférentielle, le travail thérapeutique consiste à favoriser la reprise de ces expériences qui ont été mises en échec dans les configurations intersubjectives antérieures. L'ensemble du cadre thérapeutique vise alors à favoriser la répétition des éprouvés traumatiques auxquels il est question de trouver une issue signifiante. Ce processus passe initialement par l'intériorisation des caractéristiques contenantes qui doivent composer le cadre de la thérapie à médiation, cadre sur lequel la structure signifiante et représentative pourra prendre appui. La thérapie à médiation engage donc à penser que le recours au médium favorise le travail de reprise des expériences psychiques à l'origine de la constitution du fond psychique ouvrant la voie à la fonction représentative (Brun, 2015) et à l'intériorisation des contenus (Chouvier, 2003), qui sont l'objet du travail de construction identitaire.

L'écriture est souvent envisagée sous l'angle du lien que sa pratique entretient avec le langage. En effet, la manipulation de la langue suppose un travail de mise en représentation favorable au processus de construction identitaire qui anime l'adolescence. Son rapport à la réflexivité et à la narrativité en fait un outil intéressant pour la question de l'élaboration de la construction de soi à l'adolescence. Cependant, il est également possible de concevoir l'écriture comme un véritable médium malléable : en effet, la construction d'un récit engage à des manipulations du matériel langagier en deçà du sens (la forme narrative ; les sonorités ; la rythmicité) ; et par ailleurs la production d'un texte en passe par une mobilisation du corps et de l'espace, qui dépend de la matérialité de ce qui produit l'écriture, son support (les feuilles, les matières), ses instruments (les couleurs, les types de scripteurs). Le cadre d'un atelier d'écriture complète également ces caractéristiques : la rythmicité, la fréquence, les régularités et les règles organisent les rencontres thérapeutiques et la dynamique intersubjective. Cet ensemble de spécificités de l'écriture, hors sens, participe de la fonction contenante accessible par sa pratique (Talpin, 2002). Le recours à différents supports et la manipulation des matériaux de l'écriture, à l'instar des matières malléables décrites par Anne Brun, peuvent rendre possible un travail sur le fond à partir duquel les représentations pourront prendre forme (Gibello, 2006). L'écriture peut donc servir de support de projection aux expériences non intégrables par le moi : sur le corps du texte peuvent alors prendre forme les expériences qui s'originent dans le corps du sujet, les vécus agonistiques infigurables (Brun et al., 2013), de la même manière qu'ils pourront s'actualiser dans le corps de la séance, c'est à dire au sein de la matérialité du cadre et de la relation avec le matériau ou le clinicien.

\section{Description de l'atelier à médiation par l'écriture}

Notre atelier vise à favoriser l'émergence de l'association libre (formelle et verbale) et tient compte des dynamiques transférentielles telles qu'elles sont conceptualisées dans le champ des médiations (transfert sur le cadre, sur le matériau, sur le thérapeute...). Pour ce faire, nous avons proposé un dispositif qui se différencie du cadre habituel de l'atelier d'écriture en trois temps 
(annonce de la consigne ; temps d'écriture ; temps de lecture). Notre atelier a ainsi vocation à se rapprocher davantage des dispositifs à médiations qui reposent sur la libre association. Afin de favoriser les processus associatifs et transférentiels, nous avons envisagé de laisser les adolescents libres quant au processus créateur par un aménagement spécifique du cadre. Nous avons privilégié un dispositif ouvert sur l'espace et la manipulation du matériau d'écriture et de ses consignes :

- Tout d'abord, la pièce a été aménagée de sorte à favoriser la liberté de mouvement et d'échanges : différents espaces, tables, chaises et fauteuils sont disponibles.

- $\quad$ Ensuite, le matériel mis à disposition a été choisi pour favoriser la richesse du processus créateur : feuilles de différentes tailles, matières, couleurs, stylos, crayons de couleurs, feutres, ciseaux, scotch, colle, agrafes, carnets, cartons, paper-board ...

- Enfin, nous avons envisagé les consignes d'écriture de trois manières différentes : initialement, les adolescents sont invités à faire et écrire ce qu'ils souhaitent au cours de la séance ; ensuite, le clinicien/chercheur mentionne la disponibilité du "Guide d'écriture ", constitué par le clinicien/chercheur, dans lequel sont présentées différentes consignes d'écriture, sélectionnées en fonction de leur pertinence au regard des processus de symbolisation. Enfin, le clinicien/chercheur propose aux adolescents, s'ils le souhaitent, d'aménager un espace d'écriture à plusieurs.

Le cas clinique que nous allons présenter s'inscrit dans un atelier individuel. Les données sont issues de la pratique clinique de l'un des auteurs, c'est pourquoi nous privilégierons la forme pronominale de la première personne du singulier pour décrire la situation clinique.

\section{CAS CLINIQUE}

\section{Présentation du cas : « Emilio »}

Emilio est âgé de 14 ans lorsque je le rencontre pour la première fois. Il est l'aîné d'une fratrie de trois enfants, tous placés au sein du même foyer depuis plus de six ans. Le parcours d'Emilio est marqué très tôt par les expériences de séparation : son père quitte le domicile familial peu de temps après la naissance de la plus jeune sœur de la fratrie ; il se retrouve ensuite séparé de sa mère suite à une ordonnance de placement, alors qu'il a 8 ans. Rapidement, Emilio est mis à l'écart de son frère et de sa sœur, initialement accueillis dans le même pôle de l'institution. Les professionnels motivent cette décision par la nécessité de protéger les deux plus jeunes enfants, car des jeux sexuels émergent dans la fratrie dont Emilio serait à l'initiative. En lien avec ces évènements, le jeune garçon évoque de manière durable un sentiment de culpabilité parfois diffus et s'attribue la responsabilité du placement de sa fratrie. Emilio inquiète ses éducateurs, interpellés par des comportements débordants, intensifiés en contexte scolaire : dans le groupe, Emilio peut se montrer impulsif et agressif; il a tenté de frapper ses professeurs ; il est injurieux et bagarreur, tant à l'égard de ses pairs que des adultes qui l'entourent. L'agressivité d'Emilio se manifeste également dans des menaces adressées à l'institution, portée par des recours à l'imaginaire proches des idées délirantes : il évoque des scénarios dans lesquels ses parents et lui-même sont érigés en vengeurs brutaux et armés, avec la volonté de s'en prendre à quiconque menace l'unité familiale. Ses éducateurs relatent aussi ses difficultés à rester concentré sur une tâche : Emilio " oublie vite », à tel point que les demandes du quotidien conduisent à des moments d'errances. Bien que son comportement débordant soit constant en milieu scolaire, l'attitude de Emilio peut fluctuer radicalement sur son lieu de vie : il oscille entre des moments de grande « docilité », se laisse oublier des éducateurs et de ses pairs, et d'autres plus intenses 
avec des cris, des coups et des invectives sexualisées et morbides. Par ailleurs, Emilio manifeste beaucoup d'intérêt pour des objets culturels aux thématiques crues, violentes et sexuelles : adepte du cinéma gore et pornographique, il se passionne également pour les jeux vidéo et la musique dont les spécificités sont de mettre en scène la violence.

La vie psychique et intersubjective de Emilio est donc marquée par un ensemble de ruptures : réelles et opérantes dans sa réalité environnementale, marquée par le placement et les déplacements ; dans la réalité de ses conduites, entre agression et soumission extrême ; dans le lien à l'autre, éprouvé jusqu'au point de rupture. Les difficultés rencontrées par Emilio et sa structure de placement ont mené à une prise en charge en institut thérapeutique éducatif et pédagogique (ITEP) qui a duré deux ans, en internat. Il ne retrouvait alors ses éducateurs et son lieu de vie que les week-end. L'absence de changements relevée par les équipes et l'inexistence de l'adhésion d'Emilio au dispositif ont conduit les professionnels de l'ITEP à mettre fin à leur accompagnement. De retour « à temps plein » sur la structure de placement, l'équipe me demande dans ce contexte de le rencontrer.

Emilio porte les stigmates du « mauvais objet » (Klein et Rivière, 1937) depuis son plus jeune âge : le traumatisme institutionnel, perceptible dans l'impossibilité de penser les relations fraternelles, fait ainsi écho au vécu infantile chaotique d'Emilio. La force des évènements qui se sont déroulés sur la structure de placement a eu pour effet de figer les professionnels dans une position de refus de l'intersubjectivité, favorisant l'assignation d'Emilio au rôle d'objet persécuteur. Cette posture institutionnelle émerge en miroir de l'organisation psychique de l'adolescent, qui subit le choc traumatique de son histoire familiale, impossible à élaborer par d'autres voies que celles du corps (tableau 1$)^{3}$, résultant sur des compulsions de répétions propagées dans tous les cadres institutionnels et relationnels. Cette organisation de la psyché du jeune garçon participe du tableau clinique des troubles de l'attention et du comportement (Vantalon, 2014 ; Lemoust de Lafosse et Blanc, 2016 ; Raoult, 2010). L'absence de contenance psychique chez Emilio apparait à travers la violence physique et verbale. Elle exacerbe l'externalisation des conflits internes en des conduites débordantes, caractérisées par leur propension à couper le lien à l'autre via des actes qui accentuent la proximité physique (bagarres, rixes, insultes à mises en scène sexualisées), soulignant la difficulté d'élaborer le paradoxe de l'adolescence et la nature anxiogène/structurante du lien à l'autre. Les adultes qui l'accompagnent identifient ces passages à l'acte du côté de l'expression d'une souffrance indicible, l'adolescent étant caractérisé par ses « impossibles à dire ». Ces conduites laissent entrevoir l'hémorragie pulsionnelle qui le soumet à des voies de décharges primaires, en l'absence de recours au langage à partir duquel la représentation jouerait son rôle de contenance et de liaison (Chagnon et Houssier, 2012 ; Huon et Rebourg-Roessler, 2017).

L'expérience traumatique du placement (Mayaux et al., 2016) puis de la position adolescente (Jeammet, 2001) surgit par le corps, à défaut de solution permise par la symbolisation (Marty, 2014 ; Roussillon, 1999a).

\section{Médium malléable et espace transitionnel}

Lorsque je rencontre Emilio, j'évoque avec lui la possibilité de le rencontrer initialement dans le but de faire connaissance d'une part, et pour également lui permettre de questionner ce qui pourrait lui poser problème dans son quotidien. Son comportement régresse massivement sur un versant violent : il cherche à s'extirper hors de la relation, agité comme s'il luttait contre quelque chose d'insupportable, ayant interprété ma proposition comme une obligation à laquelle

\footnotetext{
${ }^{3}$ Les tableaux 1, 2 et 3 figurent en annexe.
} 
il ne pourra se soustraire. Ces comportements contre la contrainte à laquelle il se sent soumis à cet instant s'accompagnent d'insultes morbides et pornographiques (tableau 1). Un certain temps est nécessaire pour lui permettre de s'apaiser et d'entendre qu'un tel espace n'est envisageable qu'avec son adhésion.

Dans les premiers instants de nos échanges, je m'intéresse à sa «culture ». Il m'explique qu'il aime les jeux vidéo, GTA V notamment, qu'il utilise comme un espace d'extériorisation sans limite. Chaque séance débute par un rituel similaire : il brandit à ma vue, dès son entrée dans la pièce, l'objet qui l'accompagne et qui va occuper ses mains et son regard pendant toute la rencontre. Nos échanges sont régulièrement ponctués par la musique qui émane des hautsparleurs de son téléphone. Les textes de ses chansons traitent de violence, de drogue, d'alcool et de sexe ; les arrangements musicaux sont simples et les rythmes percutants. Emilio rit de manière incontrôlée et l'évocation de la violence, dans les insultes ou dans ses fantasmes, suscite chez lui une grande excitation. Le plaisir qu'il prend au flirt d'avec les limites est perceptible dans son attitude qui m'évoque les récits de l'équipe éducative : ils décrivent un adolescent qui joue au " chat et à la souris »; nargue ses éducateurs avec des jeux-vidéo, de la musique ; l'adulte le surprend, lui rappelle les interdits, le sanctionne. À l'issue de ces séquences, l'explosion est inévitable.

En psychothérapie, l'écriture émerge spontanément car Emilio manipule tout ce qu'il peut saisir sur le bureau, notamment les feuilles et les stylos. Je lui propose de jouer avec l'écriture, et ce qui retient son attention est la consigne du « cadavre exquis ». Ce jeu rythme nos rencontres pendant plusieurs mois, au cours desquels il rivalise d'inventivité et de subversion pour produire le texte le plus dur et le plus violent possible. La perspective de créer des textes violents sur la base des interactions avec mes propres écrits suscite son hilarité et des moments de régression au cours desquels il ne contrôle ni ses textes ni l'expression de son corps.

Ces quelques premiers éléments contiennent les enjeux psychiques dans lesquels l'adolescent se trouve : l'évocation du suivi psychothérapeutique actualise chez lui des fantasmes de persécution intenses qui le renvoient à une thématique de la contrainte qui lui est insupportable. Cet épisode témoigne de la nécessité de se faire objet dans la rencontre, assez survivant pour tolérer le torrent pulsionnel, assez contenant pour sécuriser l'adolescent dans cet espace d'insécurité réactualisé, et suffisamment malléable (Mille et al., 2015 ; Roussillon, 1999a) pour donner une issue favorable à ce conflit qui se répète sur la scène transférentielle.

La rencontre est alors d'emblée marquée par les obstacles à l'engagement thérapeutique (Marteaux, 2008) tant la relation cristallise chez Emilio le caractère anxiogène du lien. Celuici s'organise alors dans un premier mouvement d'apprivoisement autour de la culture d'Emilio, de ses centres d'intérêts, dans une recherche d'interface facilitant la liaison. Au contact des objets qui mobilisent ses investissements, la scène représentationnelle est saturée en éléments de violence, de même que l'espace sonore, mobilisé tout entier par l'adolescent (tableau 1). Dans l'espace de nos rencontres, il étale sa fascination pour les thématiques et les éprouvés extrêmes, contrôle le cadre sensoriel au sein duquel il évolue, sans rencontrer de mon côté cette tentative de castration (je le laisse faire) dont je pense percevoir qu'elle lui est insurmontable.

Emilio semble également pris dans une problématique de fuite du regard de l'autre. L'intérêt porté à ses objets, à son téléphone, au monde sensoriel qu'il construit tout autour de lui, évoque la nécessité d'invoquer des objets transitionnels favorables à la tolérance de la rencontre. Le recours à ces objets semble faciliter la construction d'un espace entre dedans et dehors du bureau, dont le franchissement est rendu tolérable, et à partir duquel la fuite et l'évitement des questions du psychologue devient possible. Le recours à l'écriture m'apparaît comme un moyen de favoriser le maintien d'un échange avec lui, qui cherche par tous moyens à fuir le regard et à se réfugier dans ce monde construit sur mesure pour garder l'autre à distance de sa propre subjectivité. Pendant plusieurs mois, le « cadavre exquis » lui permet d'exprimer sa brutalité et 
de tester les limites de la relation. En effet, Emilio jubile en écrivant, à l'idée que les mots seront ensuite lus à haute voix. Par ailleurs, l'adolescent expérimente dans le même temps la possibilité de cacher les contenus qui l'envahissent, par le mouvement de replis du papier imposé par le « cadavre exquis ", et la possible coconstruction qui résulte du passage de la feuille entre nous. De cette manière, il peut par ces jeux exprimer sa violence, dans une voie dont il perçoit qu'elle est tolérée dans ce cadre spécifique, et à partir duquel une autre issue que le conflit est possible.

\section{Contrainte et contenance à l'adolescence}

Emilio accepte progressivement de nouvelles consignes d'écritures, à condition que les « cadavres exquis » inaugurent chaque nouvelle séance. À l'appui de ces consignes, Emilio expérimente de nouvelles narrations et l'émergence de thématiques plus riches qui laissent entendre l'ouverture de l'espace représentationnel. En dépit de cela, l'agir et la violence caractérisent toujours intensément les associations d'Emilio. Il s'agite sur sa chaise, déchire les feuilles de papier, les perce en même temps qu'il expulse oralement toute sa colère, à l'image des mots qu'il écrit. Emilio adopte également un langage et un comportement sexuellement marqués : il se met en scène avec les femmes qu'il côtoie, évoque des fantasmes de coït, mime des postures sexuelles. Peu à peu, il accède à la capacité à parler de ses écrits, notamment en lien avec la notion de secret et le désir de cacher au regard de ses éducateurs les textes qu'il a produits. Ainsi, un premier mouvement de réflexivité l'a mené à m'interroger sur la possibilité de conserver ses textes dans un espace dédié, entre les séances. Je lui propose de ranger la pochette dans laquelle je conserve ses textes dans une petite pièce dédiée à l'archivage de mes dossiers, fermée à clef. Le rituel se colore donc de cette demande, quasi systématique à chaque fin de séance, qui consiste à cacher ses textes (tableau 2).

Dans la verbalisation et dans l'écriture, il se laisse aller à des processus narratifs régressifs, sadiques et sexualisés : La question des failles dans le processus de symbolisation primaire (Brun, 2016) est au cœur de cette première phase du travail thérapeutique avec Emilio, tant son corps et ses mots sont imprégnés d'un caractère cru et violent, au sein desquels se loge l'énigme de la sexualité naissante et de la relation à l'autre. La notion de relation contient pour Emilio toute l'ambivalence de sa situation : l'autre est un objet étrange, à contrôler, avec qui le lien se présente comme une obligation et une contrainte en écho à l'ordonnance de placement.

Ses associations sensori-motrices font figure d'échos extériorisés et de mises en actes du caractère irréversible et destructeur des transformations subies par le moi-peau face aux angoisses archaïques de l'adolescent (Anzieu, 1987). Cette problématique semble également complexifiée par l'émergence de la pulsion sexuelle du pubertaire (Lazartigues, 2013), dont le caractère énigmatique prend forme dans les expulsions thématiques crues. Son attitude est donc marquée par les signifiants formels témoins de son insécurité, dont la portée est intensifiée par la résurgence de la pulsion sexuelle. L'écriture est d'abord investie comme un avatar de la répétition, en ce sens que ce qui se joue dans le texte agit en double de ce qui prend forme dans la matérialité du cadre (tableau 1).

Progressivement, l'agitation et la violence se transforment en quérulence, qui s'étaye sur un sentiment d'injustice et de passivité intolérable. Emilio concentre son agressivité en début de séance sur l'activité scripturale et préférentiellement sur les « cadavres exquis », puis oriente le reste du temps de la rencontre vers la verbalisation et le récit de son quotidien, de ses états internes. Emilio bénéficie alors d'un premier espace-temps contenu dans le temps de la séance pour extérioriser ses conflits de manière agie. Suite à ce premier mouvement, il devient possible pour lui de lier une narration à ses états internes, au cours de laquelle il questionne davantage sa situation d'enfant placé et la contrainte qui s'y adosse. À ce moment 
de la prise en charge, le cadre trouve une forme de contenance qui permet à Emilio la circonscription d'un espace-temps spécifique qui puisse accueillir ses angoisses archaïques. Un débordement est permis dans ce cadre, ouvrant à la possibilité de s'affranchir des limites habituellement imposées à l'écoulement pulsionnel. Emilio a pu dans ce contexte faire d'abord l'expérience d'une certaine liberté à exprimer son émoi et sa pulsionnalité là où il rencontre habituellement le choc de la castration et l'injonction à se contenir dans le discours éducatif. Cette expression par l'agir a également pris place dans un cadre à couches multiples : les jeux de « cadavres exquis » ont d'abord permis de border l'expression de la pulsion par l'écrit et la matérialité du mot, via un jeu de va-et-vient entre montrer et cacher la charge pulsionnelle au regard de l'autre (en pliant le papier sur luimême par exemple), et à l'épreuve du cadre relationnel de nos rencontres. Son intérêt pour ce type de support décrit la volonté d'Emilio de trouver une solution par lui-même à ces débordements irrépressibles dont il est l'objet et dont il semble percevoir les effets dans l'espace de la relation. Le cadre spatial a également été mobilisé dans le processus d'émergence de la fonction contenante. En écho au jeu de « cache-cache » réalisé sur la feuille de papier, un processus similaire s'est produit au sein des cadres spatiaux et temporels dans l'émergence du rituel visant à cacher ses textes aux yeux de l'extérieur dans une pièce spécifique. Emilio a ainsi opéré une mise en acte, dans le double rapport à l'écriture, entre processus de production et traitement du texte, du jeu infantile du « coucou-caché », marqueur de la découverte de l'objet et de la subjectivité.

La position d'Emilio évolue d'une dynamique narcissique destructrice du lien à l'émergence de mouvements réflexifs qui rendent possible la prise en compte dans son espace psychique de sa propre pulsionnalité. Dans cette perspective, le caractère survivant et malléable du cadre, du clinicien, du médium (Roussillon, 1999a ; Chagnon et Houssier, 2012) lui permet d'exprimer sa pulsion et d'appliquer ses propres solutions dans un contexte favorable et hors des réponses habituelles marquées par un impératif de la castration qu'il ne pouvait intérioriser. Cet ensemble de dynamiques véhiculées par les constituants du cadre thérapeutique a favorisé l'intériorisation de la fonction contenante de ce cadre. Cet ensemble de processus a également permis d'introduire Emilio à une position de réflexivité sur son propre positionnement subjectif (Chagnon et Houssier, 2012). L'usage du fond matériel (les feuilles de papier et l'espace physique de la rencontre) sur lequel il a pu focaliser sa destructivité a précédé l'émergence des représentations de contenance d'abord dans la matérialité du cadre (cacher les textes et les écrits dans une pièce spécifique), vers la représentation de la feuille de papier comme cadre de la représentation. Emilio raconte d'abord avec son corps la violence qui le harcèle (il tape, déchire, insulte); puis il accède à un mouvement de projection qui favorise un retour sur soi de sa propre expérience subjective qui devient manipulable psychiquement (replis du papier, cacher les textes et leur violence), pour enfin trouver les mots qui lui permettent de raconter son histoire. Dans ce va et vient entre les dedans et les dehors, un processus de transitionnalité s'engage : l'autorisation qui lui est faite d'explorer les espaces, celui du cadre, de l'écriture, de la relation, en projetant sur ces supports sa propre conflictualité, a permis de la rendre tolérable, de sorte à favoriser un mouvement d'intériorisation d'où peut émerger la réflexivité (tableau 2).

\section{De la représentation à la réflexivité}

À mesure que s'étayent les processus d'exploration du cadre et le déploiement de la dynamique transférentielle, apparaissent de nouvelles thématiques dans les textes de l'adolescent. Les conflits fraternels, la relation à sa mère et à ses éducateurs deviennent des champs qu'il explore de manière privilégiée. À l'occasion d'un de ses textes, qu'il réalise avec concentration, il figure une scène de bagarre fraternelle qui met en scène les causes, les 
acteurs et les conséquences de ce conflit. Sur la base de son texte, Emilio associe en évoquant la situation d'un autre adolescent accompagné par ses éducateurs (tableau 3). Nos échanges se poursuivent autour de la question de son placement, des destins des autres jeunes qu'il côtoie, le sien et celui de sa fratrie. Parler des autres enfants du foyer lui permet de rebondir sur son propre sentiment. Il dit son angoisse d'être « repéré » lorsqu'il est au collège (tableau 1). L'élaboration de ses fantasmes sert également de support aux questions posées par sa situation familiale, qui nous mènent à parler de sa mère. Avec ses récits, Emilio témoigne de son envie d'être vu autrement que comme un enfant placé, reconnu pour ses capacités et ses «bonnes » actions, ses performances scolaires.

L'épisode du conflit fraternel engage un double mouvement de l'ordre de l'expérience du miroir, qui passe par la mise à distance de l'expérience jusqu'au retour sur soi de la représentation. D'abord, Emilio décrit dans l'écriture un événement de manière factuelle et à distance de son éprouvé. Le fil associatif le mène à décrire un événement similaire survenu chez un de ses amis, également placé. L'expérience de l'autre fait office de support au déroulement de la pensée d'Emilio, sur lequel émergent l'affect et la mise en sens de la représentation. Le retour sur soi de l'expérience, marquée par le rapport à l'abandon et à la culpabilité, s'opère donc à la suite d'un mouvement d'externalisation et de projection sur l'autre. La réflexivité suit cette trajectoire dont le point de départ est la mise à l'écrit. La narration de Emilio évolue d'un récit mis en acte à travers le corps vers l'intériorisation de la fonction contenante de l'ensemble du cadre à médiation, lui permettant de déployer les processus représentatifs qui sous-tendent l'accès à la réflexivité. Nous identifions ici comment l'écriture a joué un rôle central dans ces deux processus. Le médium malléable a été mobilisé dans ses rapports au fond comme représentant de la psyché, rendant accessible l'intériorisation de la fonction contenante. En appui sur cette fonction, le rapport à la forme a vu naitre l'émergence de la capacité à extraire de soi une représentation indicible, et dont la mise en forme est rendue possible par projection sur la figure de l'autre. Mise en tension par le fil associatif et projectif, la représentation nouvellement construite peut alors être réappropriée et intériorisée pour donner impulsion au phénomène réflexif. Les dernières phases de notre suivi amorcent également de nouvelles formes de projection vers l'avenir chez Emilio, qui développe des capacités à se saisir de la temporalité comme un espace d'élaboration et d'idéalisation propice à la reconstruction de son identité. Les consignes d'écriture qui lui ont été proposées ont permis de condenser à la fois la fonction contenante de l'écriture et la fonction réflexive du psychisme sur lequel elle s'étaye, pour mobiliser un véritable processus de changement, appréciable dans le développement de la narration.

\section{DISCUSSION}

Cette présentation nous renseigne sur les éléments processuels qui peuvent prendre place dans un atelier individuel à médiation thérapeutique par l'écriture.

Tout d'abord, l'ensemble du cadre a eu une fonction d'accueil de la répétition de la conflictualité psychique portée par l'adolescent. Nous avons vu comment le cadre matériel, temporel et spatial était chargé de la tension pulsionnelle portée par le sujet. Dans un second temps, l'exploration des différentes couches des cadres a laissé émerger un travail de mise en représentation de la conflictualité. La matérialité et la spatialité du cadre ont vu apparaitre l'extériorisation des conflits psychiques (mises en actes), reportés également sur le fond et le support de l'écriture (le matériau et les instruments). L'intériorisation progressive de la fonction contenante a pu donner lieu à une écriture de ces scènes qui auparavant marquaient l'espace sonore de l'atelier, au sein d'une narration qui rompait avec la problématique de 
l'agir. Ce premier mouvement représentatif (l'écriture) semble s'originer dans la tolérance de la pulsionnalité, autorisée au sein du cadre, et être le commencement d'un processus de mise à distance, par projection sur la feuille de papier des représentations qui ont pu prendre forme dans le double espace contenant du cadre et du support. Emilio a alors pu faire l'expérience de la réflexivité en symbolisant son propre fonctionnement.

Cet exposé clinique donne plusieurs indications sur l'usage d'une médiation par l'écriture et sur la manière dont elle mobilise un adolescent aux prises avec sa problématique de placement et celle de l'adolescence, quand la parole ne trouve pas à se déployer et mène à des recours au corps (Marty, 2014 ; Roussillon, 1999a). Nous avons voulu montrer que la médiation thérapeutique permet de reconnecter le sujet à son objet pour favoriser une reconnexion à soi-même (Roussillon, 2014). En effet, Emilio construit une dynamique créatrice à partir d'un mouvement d'indifférenciation entre sujet et objet, progressivement rendue tolérable par l'intériorisation de la fonction contenante, et dont la tolérance autorise les mouvements de projection et de différenciation sujet-objet à partir desquels la réflexivité peut émerger, par le retour sur soi des représentations projetées à l'extérieur.

D'autre part, nous avons souhaité mettre en évidence les particularités du medium « écriture "), et la façon dont il met en jeu plusieurs niveaux de symbolisation, de l'archaïque à la construction adolescente et son articulation entre le corps et la psyché. En cela, ce travail fait écho aux recherches de Viardot et al. (2016), qui nous montrent comment la pratique de l'écriture par des adolescents mène à la mise en récit de soi et de ses éprouvés. Nous repérons aussi ce que Engasser (2014) avait noté auprès d'une population tout à fait différente : l'écriture permet de déplacer l'excitation du corps vers « le corps du texte ». En effet, les éléments matériels du support d'écriture portent, au même titre que les autres éléments du cadre, une fonction contenante qui participe du processus d'extériorisation-intériorisation de la représentation. C'est donc en appui sur l'articulation de ces divers degrés de cadre que le processus se déroule. Par ailleurs, une analyse plus attentive des thématiques de l'écriture rend observables les phénomènes représentatifs et les conflits psychiques de l'adolescent et de sa situation de placement (problématiques de séparation, d'autonomisation, familiales, environnementales). Nos observations soutiennent ainsi les travaux qui décrivent la capacité de l'écriture à favoriser la construction de l'identité et l'accès à l'autonomisation de l'adolescent (Cavin-Picard, 2007 ; Viardot et al., 2016, 2017) d'une part, et la tentative de mise en sens et en récit de l'histoire subjective (Cavin-Picard, 2015 ; Chiantaretto, 2014 ; Chidiac, 2008, 2013).

\section{CONCLUSION}

La mise en perspective de données associant les problématiques qui touchent les adolescents en situation de placement et la délinquance des mineurs appelle la nécessaire question de la prise en charge et de l'accompagnement. En effet, alors que certains rapports soulignent les liens entre parcours de placement, troubles des conduites avec passages à l'acte violent et protection judiciaire de la jeunesse (PJJ) (McGuigan et al., 2018), d'autres notent également que les jeunes accompagnés dans le cadre de la PJJ sont plus impactés par les processus de déscolarisation, de ruptures des liens sociaux, les fugues, les symptômes dépressifs et suicidaires (Botbol et al., 2010) manifestes d'un mal-être. Les adolescents placés qui manifestent des troubles du comportement doivent, dans cette perspective, faire l'objet d'une attention particulière tant leur situation est susceptible de déboucher sur une problématique psychopathologique, judiciaire et marginalisante.

La littérature, cependant, met en exergue la complexité liée à l'évaluation des effets thérapeutiques issus de la prise en charge des sujets placés, bien qu'ils soient massivement 
représentés dans les institutions de soins. L'absence d'étayage familial, les difficultés de coordination entre les services sanitaires et sociaux, l'efficacité toute relative des mesures de prévention auprès de ces populations, sont identifiés comme des facteurs favorisant l'insuffisance des offres de soin (Kayser et al., 2011).

Cet exposé nous engage ainsi à poursuivre les démarches d'exploration des dispositifs thérapeutiques adaptés à cette population, et également à nous intéresser de plus près à l'évaluation des dispositifs cliniques proposés aux adolescents en situation de placement. Dans une recherche récente, Brun, Attigui et Roussillon (2016) ont entrepris de proposer des dispositifs qui puissent permettre l'évaluation des médiations thérapeutiques. Il peut donc s'avérer intéressant de poursuivre ces questionnements à l'appui de la médiation par l'écriture, proposée à des adolescents en situation de placement.

\section{RÉFÉRENCES}

Anzieu D., Anzieu A., Guillaumin J., Houzel D., Lecourt É., Enriquez E., et al. Les enveloppes psychiques, Paris, Dunod, 1987.

Aulagnier P., La violence de l'interprétation, Paris, Presses Universitaires de France, 1975.

Bion WR., Réflexion faite, Presses Universitaires de France, 1983.

Bion WR., Aux sources de l'expérience. Paris, Presses universitaires de France, 1979.

Birraux A., «Les adieux à l'enfance », in Moro MR. (dir.) Devenir adulte, Paris, Armand Colin, 2013.

Botbol M., Choquet, LH., Grousset, J., « Éduquer et soigner les adolescents difficiles », in Neuropsychiatrie de l'Enfance et de l'Adolescence, 2010, pp. 224-233.

Braconnier A., «Construction des liens et continuité des soins à l'adolescence», in Neuropsychiatrie de l'Enfance et de l'Adolescence, 2009, pp. 464-467.

Brun A., «Médiation thérapeutique picturale et associativité formelle dans les dispositifs pour enfants avec troubles envahissants du développement ", in La psychiatrie de l'enfant, 2014, pp. 437-64.

Brun A., « La rencontre analytique dans les dispositifs à médiations thérapeutiques, aux limites de l'analyse », in Revista Latinoamericana de Psicopatologia Fundamental, 2015, pp. 234-52.

Brun A., « Spécificités de la symbolisation dans les médiations thérapeutiques », in Cliniques, 2016, pp. 16-44.

Brun A., Chouvier B., Roussillon R., Manuel des médiations thérapeutiques, Paris, Dunod, 2013.

Brun A., Roussillon R., Attigui, P., Évaluation clinique des psychothérapies psychanalytiques, Paris, Dunod, 2016.

Cavin Piccard M-C., «L'atelier d'écriture, un outil pour développer le processus d'autonomie chez des adolescents en rupture », in Thérapie Familiale, 2007, pp. 523-531.

Cavin Piccard M-C., «Appartenance et quête identitaire par le chemin de l'imaginaire et de la créativité dans des groupes d'écriture », in Thérapie Familiale, 2015, pp. 187-200.

Chabert C., Les séparations, Paris, Eres, 2013.

Chagnon J-Y., Houssier F., « L'illusoire attente de la demande », in Adolescence, 2012, pp. 919-933.

Chatagner A., Olliac B., Choquet L-H., Botbol M., Raynaud J-P., « Adolescents reçus en urgence en psychiatrie infanto-juvénile ", in Neuropsychiatrie de l'Enfance et de l'Adolescence, 2015, pp. 124-32.

Chiantaretto J-F., « S'écrire: survivre ou se faire naitre dans le regard de l'autre? », in Le Coq-héron, 2014, pp. 46-49.

Chidiac N., «Écriture du traumatisme ou traumatisme de l'écriture ? », in Annales Médico-psychologiques, 2008, pp. 308-14.

Chidiac N., Ateliers d'écriture thérapeutique, Elsevier Masson, 2013.

Chouvier B., « Objet médiateur et groupalité », in Revue de psychothérapie psychanalytique de groupe, 2003, pp. $15-27$.

Corcos M., « Accueil des adolescents : engagement et créativité ». In Annales Médico-psychologiques, 2007, pp. 723-8.

Derivois D., «La traversée traumatique de l'enfant placé en institution : un point de vue clinique ». In Pratiques Psychologiques, 2008, pp. 339-56. 
Drieu D., Sarabian S., Plagès M., « Les adolescents vulnérables et les alternatives thérapeutiques en protection de l'enfance », in L'Évolution Psychiatrique, 2012, pp. 265-77.

Engasser O., « La mise en œuvre d'un atelier d'écriture avec des patients déments au stade sévère : fondements, objectifs et résultats empiriques », in Revue de psychothérapie psychanalytique de groupe, 2014, pp. 181193.

Gibello B., « La question du fond et de la forme des représentations mentales », in Le Journal des psychologues, 2006, pp. 43-48.

Golse B., «Les signifiants formels comme un lointain écho du bébé que nous avons été », in Le Carnet PSY, 2007 , pp. 39-47.

Green A., Le travail du négatif, Ed. de Minuit, 1993.

Haag G., « Réflexions de psychothérapeutes de formation psychanalytique s'occupant de sujets avec autisme »,

Revue française de psychosomatique, 2005, pp. 113-24.

Hanifi M., « Le couple Inhibition/Agir à l'adolescence : du symptôme à la fonction », in Psychothérapies, 2019, pp. 9-14.

Houssier F., « Transgression et recours à l'acte à l'adolescence : une forme agie d'appel à l'objet », in Annales Médico-psychologiques, 2008, pp. 711-6.

Huon P., Rebourg Roesler, C., « Psychopathologie de l'énonciation dans le champ de la clinique de l'agir », in L'Évolution Psychiatrique, 2017, pp. 255-71.

Jeammet P., « Innovations en clinique et psychopathologie de l'adolescence », In Annales Médicopsychologiques, 2001. pp. 672-678.

Jeammet P., "La violence à l'adolescence : une défense identitaire », In Neuropsychiatrie de l'enfance et de l'adolescence, 2002, pp. 434-441.

Jeammet P., « Entre soi et non-soi, l'espace de la rencontre », in L'Évolution Psychiatrique, 2019, pp. 127-38.

Kayser C., Jaunay E., Giannitelli M., Deniau E., Brunelle J., Bonnot O., et al. « Facteurs de risque psychosociaux et troubles psychiatriques des jeunes pris en charge par l'aide sociale à l'enfance et ayant recours à des soins hospitaliers », in Neuropsychiatrie de l'Enfance et de l'Adolescence, pp. 393-403.

Lazartigues A., « De l'enfance à l'âge adulte : quelques effets d'un environnement postmoderne sur le processus de subjectivation », in Neuropsychiatrie de l'Enfance et de l'Adolescence, 2013, pp. 302-9.

Lemoust de Lafosse H., Blanc R., «Étude sur l'attachement et les troubles du comportement à l'adolescence chez des jeunes placés en institution », in Neuropsychiatrie de l'Enfance et de l'Adolescence, 2016, pp. 93-101.

Marteaux A., «Soi est un autre : construction et déconstruction identitaires à l'adolescence », in Cahiers critiques de thérapie familiale et de pratiques de réseaux, 2008, pp. 183-198.

Marty F., «Sortir de l'adolescence », In Moro MR. (dir.) Devenir adulte : chances et difficultés, Paris, Armand Colin, 2014.

Mayaux F-X., Viodé C., Derivois D., « La dynamique relationnelle entre l'éducateur et l'enfant en contexte de placement familial », in Annales Médico-psychologiques, 2016, pp. 183-8.

McGuigan WM., Luchette JA., Atterholt R., « Physical neglect in childhood as a predictor of violent behavior in adolescent males », in Child Abuse \& Neglect, 2018, pp. 395-400.

Mille C., Barthe E., Bon Saint Come M., Delhaye M., « Thérapies avec médiations, ou la thérapeutique par surcroît ", in Neuropsychiatrie de l'Enfance et de l'Adolescence, 2015, pp. 332-40.

Milner M. « Le rôle de l'illusion dans la formation du symbole », in Revue Française de Psychanalyse, 1955, pp. 841-874.

O’Neill M., Risley-Curtiss C., Ayón C., Williams LR., « Placement stability in the context of child development ", in Children and Youth Services Review, 2012, pp. 1251-8.

Rabeyron T., « Les processus de symbolisation et de représentation comme espace transitionnel pour la psychanalyse et les neurosciences », in L'Évolution Psychiatrique, 2016, pp. 160-75.

Raoult PA., « Les désétayages psychiques dans les agirs adolescents », in L'Évolution Psychiatrique, 2010, pp. 304-26.

Klein M., Riviere J., L'amour et la haine, Paris, Petite Bibliothèque Payot, 1937.

Roussillon R., Agonie, clivage et symbolisation, Paris, Presses Universitaires de France,1999a.

Roussillon R., « Les enjeux de la symbolisation à l'adolescence », in Adolescence, 1999b, pp. 7-23.

Roussillon R., « Le transitionnel et l'indéterminé ». In : Chouvier B. (dir.) Les processus psychiques de la médiation, Paris, Dunod, 2003.

Roussillon R., Manuel de psychologie et de psychopathologie clinique générale, Issy-Les-Moulineaux, Elsevier Masson, 2014.

Talpin J-M., « Des fractures du moi au je d'écriture : écriture, médiation et intégration psychique », In Chouvier B. (dir.) Les processus psychiques de la médiation, Paris, Dunod, 2002. 
Vantalon V., « Expression phénotypique du TDAH en fonction de l'âge », in Annales Médico-psychologiques, revue psychiatrique, 2014, pp. 287-92.

Viardot C., Titia-Rizzi A., Lachal J., Moro MR., « Groupe d'écriture thérapeutique pour patients hospitalisés dans une maison des adolescents », in La Psychiatrie de l'Enfant, 2016, pp. 173-207.

Vitte L., Belloncle V., Gérardin P., « Adolescents hospitalisés dans les services de psychiatrie adulte », in Neuropsychiatrie de l'Enfance et de l'Adolescence, 2017, pp. 360-7.

Winnicott DW., La capacité d'être seul, Paris, Payot, 1958.

Winnicott DW., La crainte de l'effondrement et autres situations cliniques, Paris, Gallimard, 1971.

Corentin Boulay

Laboratoire Interpsy, EA 4432

Université de Lorraine

Campus Lettres et Sciences Humaines et Sociales

23, Bd Albert $1^{\text {er }}$

54015 Nancy cedex, France corentin.boulay@univ-lorraine.fr 